\title{
Developing an implicit measure of disgust propensity and disgust sensitivity: Examining the role of implicit disgust propensity and sensitivity in obsessive-compulsive tendencies
}

\author{
Emma Nicholson*, Dermot Barnes-Holmes \\ Department of Psychology, National University of Ireland, Maynooth, Co. Kildare, Ireland
}

\section{A R T I C L E I N F O}

\section{Article history:}

Received 10 October 2011

Received in revised form

27 January 2012

Accepted 8 February 2012

\section{Keywords:}

Disgust propensity

Disgust sensitivity

IRAP

Obsessive-compulsive tendencies

\begin{abstract}
A B S T R A C T
Background and objectives: There is increasing evidence that disgust responding occurs at both a primary and secondary level in the form of disgust propensity and disgust sensitivity. The unique contributions of anxiety and disgust need to be established if disgust is to be implicated in the etiology of anxiety disorders such as obsessive-compulsive disorder (OCD). The primary objective of the current study was to develop two separate implicit measures of disgust propensity and sensitivity and to explicate the role of implicit disgust propensity and sensitivity in avoidance behavior and OC tendencies.

Methods: The current study ( $N=33$ undergraduate students) utilized a measure of implicit cognition, the Implicit Relational Assessment Procedure (IRAP), to independently analyze disgust propensity and disgust sensitivity. In addition, a series of behavioral approach tasks (BAT) and questionnaires measuring general disgust, obsessive-compulsive $(\mathrm{OC})$ tendencies and general psychopathology were implemented to validate the implicit measures.

Results: Disgust sensitivity predicted avoidance behavior on the BATs independent of disgust propensity and anxiety, while disgust propensity did not. Both disgust propensity and sensitivity predicted selfreported $\mathrm{OC}$ tendencies and individually predicted obsessing and washing concerns, respectively. Limitations: Our findings are based on a non-clinical student sample and further research is required for generalization to OCD.

Conclusions: The implicit measures appeared to be measuring two separate constructs and had differential relationships with behavior and $O C$ tendencies. Overall, the results support current theories relating to pathological disgust and $O C D$.
\end{abstract}

(c) 2012 Elsevier Ltd. All rights reserved.

\section{Introduction}

Disgust is a universally acknowledged negative emotion encompassing physiological, cognitive and behavioral domains (Davey, MacDonald, \& Brierley, 2008; Oaten, Stevenson, \& Case, 2009). Early theorists treated disgust as repulsion at oral incorporation, that is, it primarily centered on food-related disgust (Haidt, McCauley, \& Rozin, 1994). Current research indicates that many other experiences may elicit disgust including body-envelope violations, animal-related, body-products and socio-moral disgust (Haidt et al., 1994). Additionally, disgust responding is said to follow two laws of sympathetic magic: 1) the law of contagion which holds that there is a permanent transfer of properties from one object to another, 2) the law of similarity which posits that objects

\footnotetext{
* Corresponding author. Tel.: +3531708 6086; fax: +35317084767.

E-mail address: Emma.Nicholson@nuim.ie (E. Nicholson).
}

which resemble one another share the same properties (Rozin, Millman, \& Nemeroff, 1986).

The last decade has seen a dramatic increase in disgust-related research, with the majority of this research conceptualizing disgust as a unitary response. Recent evidence, however, suggests that disgust can be separated into two constituents, propensity and sensitivity. Disgust propensity is an individual's tendency to experience disgust while disgust sensitivity is how negatively the individual appraises their experience of disgust (van Overveld, de Jong, Peters, Cavanagh, \& Davey, 2006). The study of both constructs is relevant in that it may be useful to measure both how easily disgusted an individual becomes, and how negatively this feeling is then appraised (van Overveld et al., 2006). Until recently, disgust sensitivity (i.e., the secondary appraisal of the initial feeling of disgust) has been underplayed in the literature with most of the research focusing on disgust propensity.

Teachman and Saporito (2009) argued that, based on cognitive models of anxiety, irrational disgust appraisals will likely be 
present if disgust's contribution to the etiology of psychopathology parallels that of anxiety's contribution, but many open questions remain. More specifically, the role of disgust in the etiology of anxiety disorders such as obsessive-compulsive disorder (OCD) has been identified as an important area for future research (Olatunji, Cisler, McKay, \& Phillips, 2010).

Obsessive-compulsive disorder is characterized by recurrent obsessions or compulsions such as washing or checking, which result in impaired social and occupational functioning (Wahl et al., 2010). Davey (2003) posited that in order to determine the extent of a relationship between disgust and any psychopathology the mediating effect of anxiety on the relationship needs to be established. Thus, it is vital that current levels of anxiety be taken into account when examining this relationship. Moretz and McKay (2008) found a direct relationship between a self-reported predisposition to become disgusted (i.e., disgust propensity ${ }^{1}$ ) and OCD contamination symptoms above and beyond anxiety. Similarly, disgust has been shown to predict general OCD symptoms and washing concerns independently of anxiety and act as an intervening variable between anxiety and spider fears, blood-injuryinjection (BII) fears and washing concerns (Olatunji et al., 2007).

In recent years, some researchers have begun to employ so called implicit measures of cognition in the study of psychopathology and anxiety (De Houwer, 2002). Implicit measures have been described as a means of assessing mental content, often in the absence of conscious recognition between this content and the response (Nosek \& Greenwald, 2009). The ego-dystonic nature of anxious phenomenon such as obsessions suggest that they are involuntary or automatic and this apparent lack of conscious control over thoughts and feelings that characterizes many psychopathologies supports the use of implicit measures in this domain (see Wiers, Teachman, \& De Houwer, 2007, for a full appreciation). According to McNally (1995), at least one type of cognitive bias encompasses each of the anxiety disorders (e.g., attentional or interpretational biases). Problematic disgust responding appears to be as a result of an information processing bias (e.g., If it gets all over me, I don't think I could cope) thus it may share some of the features of automaticity. The involuntary nature of this biased processing of environmental cues is viewed as the trademark of automaticity in anxiety (McNally, 1995; Teachman, 2007; Wiers et al., 2007). As such, it is important to examine these biases at both the implicit and explicit level in order to attain a greater understanding of the etiology and preservation of psychological conditions such as OCD (Wiers et al., 2007).

The Implicit Association Test (IAT) has been utilized to measure general disgust in relation to spider and snake fear and general disgust (e.g., Teachman, Gregg, \& Woody, 2001, Huijding \& de Jong, 2007; Zinkernagel, Hofman, Dislich, Gschwendner, \& Schmitt, 2011). Critically, the stimuli used in these studies (e.g., disgusting, gross, repulsive, dirty) made it likely that the IATs were targeting primary disgust reactions - that is, disgust propensity. In relation to measuring disgust sensitivity, the methodology of the IAT gives rise to difficulties because disgust sensitivity involves appraisal of an initial feeling and it has been argued that the IAT cannot accommodate the measurement of such complex conditional beliefs (De Houwer, 2002). Importantly, in the context of the current study, a relatively new methodology known as the Implicit Relational Assessment Procedure (IRAP; Barnes-Holmes et al., 2006; see Nosek, Hawkins, \& Frazier, 2011) appears to offer a way

\footnotetext{
${ }^{1}$ Moretz and McKay (2008) defined disgust sensitivity as "the trait-like predisposition of a person to become disgusted" (p.p.707). In keeping with the definitions set out by van Overveld et al. (2006), this would be conceptualized as disgust propensity in the current context in that appraisals have no role.
}

of measuring conditional beliefs, at the implicit level (see Hughes, Barnes-Holmes, \& De Houwer, 2011). It has been argued recently that even propositional processes may possess certain features of automaticity, and thus the propositional nature of the IRAP does not, ipso facto, undermine the claim that it is tapping into automatic responses (see Hughes et al., 2011, for a detailed discussion). Furthermore, the recently offered Relational Elaboration and Coherence model (REC; Barnes-Holmes, Barnes-Holmes, Stewart, \& Boles, 2010), which underpins the IRAP, assumes that automatic and strategic responses sit at opposite ends of a continuum rather constituting separate or dichotomous psychological processes (see below). As such, the IRAP should allow for the measurement of not only disgust propensity but also sensitivity, even though the latter may be a less automatic, or slightly more controlled aspect of disgust responding, than the former.

A second limitation of the IAT is that the belief under scrutiny is only measured as a function of its relation to the opposing category inserted into the IAT. That is, it provides only a relative measure of implicit cognition (De Houwer, 2003). For instance, in the Teachman et al. (2001) study faster responding to spider-positive and snake-negative than to the opposite pattern (i.e., spidernegative and snake-positive) could be interpreted in various ways. Participants could (a) like spiders and dislike snakes, (b) they could dislike spiders and snakes, but the latter are disliked more than the former or (c) they could like spiders and snakes, but the former are liked more than the latter. This disadvantage is particularly relevant to the study of constructs such as spider fear and disgust as they have no generally accepted dichotomous relationship with another construct to provide an appropriate contrasting category (Teachman, 2007). The IRAP, on the other hand, aims to provide a non-relative measure of implicit attitudes by allowing for the assessment of a single target, irrespective of the chosen opposing category (see Nicholson \& Barnes-Holmes, in press, for empirical support for this claim).

The IRAP is a computer based procedure which requires participants to respond quickly and accurately in a manner that is consistent or inconsistent with their previous learning history. The fundamental hypothesis is that responding should be quicker and more accurate on bias-consistent rather than bias-inconsistent trials. In relation to anxiety, the IRAP has successfully measured an anti-spider bias and predicted avoidance of a live spider (Nicholson \& Barnes-Holmes, in press). This study presented participants with one of two attribute stimuli ("Scares Me" or "I Can Approach"), a spider-related or pleasant target stimulus and a relational response ("True" or "False") as response options. Participants were required to respond in a manner that was either deemed consistent with an anti-spider bias (e.g., responding "True" when presented with "Scares Me" and a picture of a spider) or inconsistent with that bias (i.e., choosing "False," given "Disgusts Me" and a picture of a spider). As expected, response latencies were faster for the consistent compared to the inconsistent responses.

The IRAP was derived from a modern behavior-analytic account of human language and cognition called Relational Frame Theory (RFT; see Hayes, Barnes-Holmes, \& Roche, 2001). The basic assumption of RFT is that the fundamental components of human language and cognition are relational, and thus the IRAP focuses on stimulus relations or propositions rather than stimulus pairings or associations (e.g., Hughes et al., 2011). The basic IRAP effect, that responding should be quicker on bias-consistent relative to biasinconsistent trials, has been explained in terms of the REC model (Barnes-Holmes et al., 2010). The REC model assumes that brief and immediate relational responses (BIRRs) will occur on most trials of the IRAP before a participant presses a response key. These responses will be based on historical and existing contextual variables, with the most likely response being emitted first (Barnes- 
Holmes et al., 2010). Based on this view, BIRRs provide the foundation for what have been commonly termed implicit attitudes (Hughes et al., 2011). On balance, BIRRs are seen as lying on a continuum of "implicitness" or automaticity, rather than constituting a discreet associative process that is completely separate or independent from controlled processing (see Hughes et al., 2011, for a detailed treatment of this issue).

The primary aim of current study was to develop implicit measures of disgust propensity and sensitivity using the IRAP. Two separate IRAPs were developed, one to measure disgust propensity and another to measure disgust sensitivity. The IRAPs presented identical pictorial stimuli depicting either disgusting or pleasant images. The differences between the two IRAPs were the worded reactions to the pictorial stimuli, which represented either disgust propensity (i.e., primary reactions such as "I am Disgusted") or disgust sensitivity (i.e., secondary appraisals such as "I Worry I'll get Sick"). A series of behavioral approach tasks encompassing the disgust domains of food-related disgust, socio-moral disgust, bodyenvelope violations and animal-related disgust were used to validate the implicit measures. Additionally, a series of questionnaires were implemented to measure general disgust, $\mathrm{OC}$ tendencies and general psychopathology. Given that this was the first study to attempt to use implicit measures to provide independent assessments of disgust propensity and sensitivity, we refrained from making specific predictions. However, due to the automatic nature of disgust propensity, it was predicted that this construct would have a closer relationship with the initial elements of disgust responding such as automatic negative thoughts (i.e., initial covert reactions). On balance, based on work by Teachman (2006), in which she argued that secondary disgust reactions would focus on beliefs about the perceived ability to cope with being disgusted (e.g., "If this gets all over me, I'll never feel clean again"), it was hypothesized that the disgust sensitivity IRAP would be a greater predictor of the behavioral aspect of disgust responding (i.e., the compulsion to hand wash).

\section{Method}

\subsection{Participants}

Participants $(N=33)$ were selected from the student population of NUI Maynooth. There were no selection criteria in order to take part in this study in relation to levels of disgust or OC tendencies. There is increasing support for the idea that OCD symptoms originate in normal human processing. Therefore, the use of nonpatient samples that score high on self-report measures of OCD may be relevant to understanding the development of OCD (see Burns, Formea, Koertege, \& Sternberger, 1995). There were 12 men and 21 women with ages ranging from 18 to 25 with a mean age of 19.73. Each participant provided written informed consent prior to taking part in the study and completed the experiment individually in the Department of Psychology at NUI Maynooth.

\subsection{Materials}

\subsubsection{Disgust Scale-Revised (DS-R; Haidt et al., 1994)}

The Disgust Scale is a 32 item scale and is frequently used to measure disgust propensity ${ }^{2}$ across seven domains of disgust

\footnotetext{
${ }^{2}$ In the original paper by Haidt et al. (1994), the DS-R was said to be measuring disgust sensitivity, but an anonymous reviewer pointed out that van Overveld et al. (2011) argued that it may be more a measure of disgust propensity. In any case, the scale was created at a time when disgust was not conceptualized as two constructs, and thus caution will be required when interpreting results arising from the DS-R.
}

including food, animals, body-products, death, body-envelope violation, hygiene and sex (van Overveld, de Jong, Peters, \& Schouten, 2011). The scale has been found to have convergent and discriminant validity and has moderate correlations with a sensation-seeking scale $(r=-.46)$ and a fear of death scale $(r=.39)$.

\subsubsection{Obsessive-Compulsive Inventory-Revised (OCI-R; Foa et al., 2002)}

The OCI-R is an 18 item self-report measure of symptoms of obsessive-compulsive disorder and was used to measure $O C$ tendencies. It has successfully differentiated between individuals with and without OCD. For a non-anxious sample, it has demonstrated good-excellent internal consistency ( $\geq .72)$, and test-retest reliability (.57-.87).

\subsubsection{Depression, Anxiety and Stress Scale-21 (DASS-21; Lovibond E' Lovibond, 1993)}

The DASS is a 21 item self-report questionnaire which covers a range of core symptoms of anxiety, depression and stress. For a non-clinical sample, it has demonstrated excellent internal consistencies among its three subscales (.82-.90), good convergent and discriminant validity $(.70-.72)$ and adequate reliability (.90-.95) (Henry \& Crawford, 2005).

\subsubsection{Implicit Relational Assessment Procedure (IRAP)}

Two IRAPs were completed by each participant (for a detailed description of the generic IRAP method see Barnes-Holmes et al. 2010). The Disgust Propensity IRAP (hereafter referred to as the DP-IRAP) presented one of two target stimuli on each trial, a Disgust response (e.g., "I Am Disgusted") or a Positive response (e.g., "I Like it"). The Disgust Sensitivity IRAP (hereafter referred to as the DSIRAP) presented a Distress appraisal response (e.g., "I Need to Look Away") or a Non-Distress appraisal response (e.g., "I Know I Won't Get Sick") on each trial. The label stimuli presented in both IRAPs were identical and consisted of one of sixteen digital images; eight were color photographs of things which would evoke disgust and the other eight were color pictures of pleasant things. All but one of the stimuli was taken from the International Affective Picture System $^{3}$ (IAPS; Lang, Bradley, \& Cuthbert, 1996). The pictorial stimuli were chosen because they reflected a range of disgust domains (e.g., animal, body-envelope violations, socio-moral etc) and the worded stimuli were based on phrases used in self-report scales which have been shown to provide separate measures of disgust propensity and sensitivity. Finally, two response options, "True" and "False" were also presented in both IRAPs (see Fig. 1).

\subsection{Procedure}

\subsubsection{Behavioral approach tasks}

Three of the behavioral approach tasks (tarantula, poop cookie, surgery) were selected because they have been used in previous studies assessing disgust (e.g., Teachman \& Saporito, 2009) and capture a range of disgust responding in a variety of domains (e.g., animal-related, food, blood-injury-injection). The socio-moral task was designed specifically for the current study as a means to measure socio-moral disgust in an approach-type manner (see Appendix A). ${ }^{4}$

\footnotetext{
3 The picture numbers from the IAPs were as follows: 1111, 1205, 1280, 3250 $9300,9373,9405,1440,1463,1710,1750,5201,5250,5731,5760$. The 16 th pictorial stimulus was a photograph of Hitler which aimed to evoke socio-moral disgust.

${ }^{4}$ A fifth control task adapted from Gordan and Teachman (2008) was used to measure anxiety, however, preliminary analysis found no relationship with selfreported anxiety. As such, it was eliminated from the final analyses.
} 

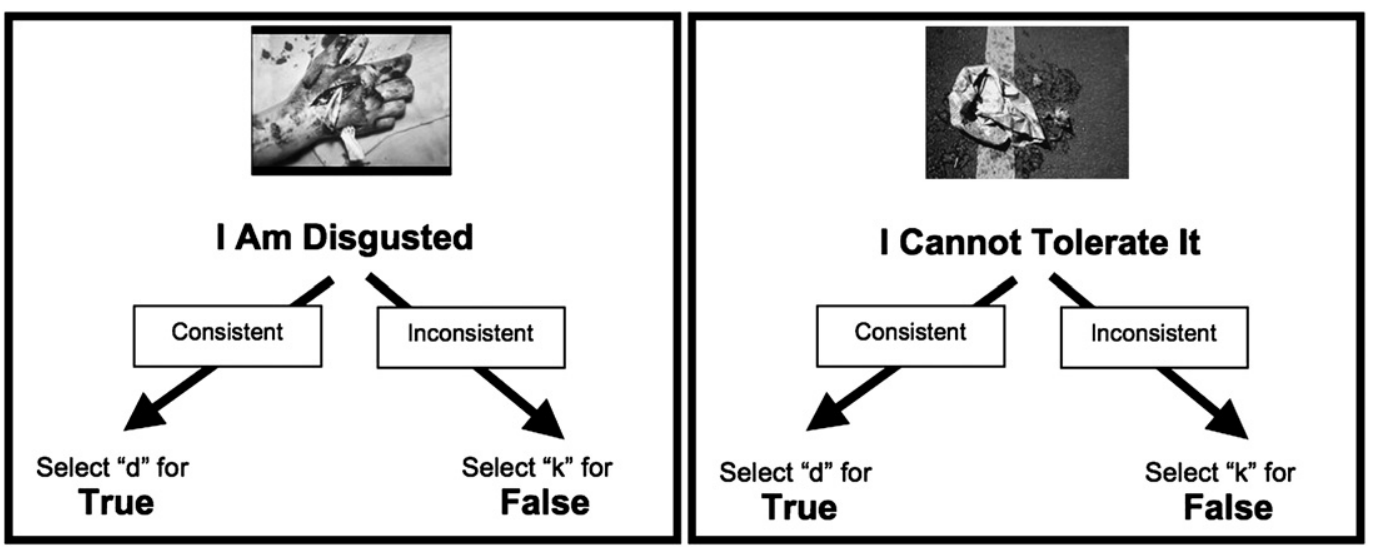

Fig. 1. Example of the Disgust-Bad and Disgust-Distressing trial-types from the DP- and DS-IRAPs, respectively.

\begin{abstract}
2.3.1.1. Tarantula skin. The molt of a tarantula of approximately 4 inches in length was placed in a small container at the end of a room. Participants were told what was in the room (the molt of a tarantula, not a live spider) and were asked if they would allow the experimenter to open the door to the room (step 1). They were asked if they were willing to enter the room (step 2), approach the spider skin as closely as they felt comfortable (step 3), ultimately picking the spider skin up (step 4) and holding it for up to or for $1 \mathrm{~min}$ (steps 5 and 6). They were allotted a score from 0 to 5 based on their performance on the task.
\end{abstract}

2.3.1.2. Surgery video (adapted from Rozin, Haidt, McCauley, Dunlop, $\mathcal{E}$ Ashmore, 1999). A video of a hip replacement surgery which became progressively more graphic was played on a computer screen to the participants. The video was $3 \mathrm{~min}$ in length. Participants were told to keep their eyes on the screen and if they wished to cease watching the video they were instructed to push a designated button on the keyboard which would stop the video. Steps completed were scored based on the amount of time spent watching the video and ranged from 0 to 5 (corresponding to six $30 \mathrm{~s}$ increments).

2.3.1.3. Poop cookie (adapted from Teachman E Saporito, 2009). A piece of chocolate that was designed to look like animal feces was placed in a litter box-shaped container filled with oats which resembled cat litter. Participants were told that it was not real feces and were asked how willing they were to step closer to the cookie (step 1). They were then asked would they be willing to stand over the container (step 2), pick up the cookie (step 3), examine it carefully (step 4), put the cookie to their lips (step 5) and step 6 involved taking a bite of the cookie. They were allotted a score between 0 and 5 based on their performance.

2.3.1.4. Socio-moral task. This task was designed to measure the extent to which participants avoid thoughts about performing socially immoral acts. Participants were first asked to think of up to three moral violations that they would consider as being weak (i.e., an act or scenario that the participant believes to be less immoral than other acts such as murder but still immoral in some sense). They were then instructed to rate how they felt about doing this task in the domains of level of difficulty, how morally uncomfortable they felt while doing the task and their willingness to do the task again. The same procedure was repeated a further three times encompassing different levels of morality; moderate, strong and extreme. The task was scored from 0 to 5 and participants were allotted one point for every two moral violations they wrote. The task acted as a behavioral approach task in that it was designed to require participants to carry out a task in stages while also inducing discomfort. It was based on the cognitive-behavioral theory of intrusive thoughts set forth by Salkovskis (1985) in which the belief that having a thought about an action is as bad as performing the action (see Shafran \& Rachman, 2004, for a review of Thought-Action Fusion).

\subsubsection{IRAP}

The IRAP is a computer based procedure that requires participants to respond quickly and accurately to blocks of trials that are consistent or inconsistent with their own beliefs. The primary datum from the IRAP is response latency, which is defined as the time in milliseconds that elapses from the onset of a trial to the emission of a correct response. A correct response on any trial was determined by whether or not the participant was completing a block of trials designed to be consistent or inconsistent with high levels of disgust propensity or sensitivity (see Fig. 1). On both IRAPs, consistent trials were those which required participants to respond in an anti-disgust and pro-pleasant manner (e.g., responding "True" when presented with a disgusting picture and "I Feel Repulsed" or "I Fear Contamination") while inconsistent required the opposite response pattern (i.e., pro-disgust and anti-pleasant; e.g., "True" when presented with a disgusting picture and "I Like It" or "I Feel in Control"). The fundamental hypothesis is that average response latencies should be shorter for blocks of consistent relative to inconsistent trials. The extent of the observed difference between the trials is assumed to provide an index of the strength of the response bias under scrutiny.

The IRAP was presented in blocks of 32 trials. There were a number of practice blocks that each participant completed in order to ensure an accuracy rate of $75 \%$ and a response latency of less than or equal to $2000 \mathrm{~ms}$ for the DP-IRAP and $2500 \mathrm{~ms}$ for the DS-IRAP. The participants were required to meet these criteria across a pair of practice blocks, within six or less blocks, before proceeding to the fixed set of six test blocks. The instructions for the IRAP were presented on paper and were read through with the experimenter to ensure that each participant understood the nature of the experiment and what was being asked of them. The experimenter stressed the importance of speed and accuracy in the IRAP. Each participant was aware that, at times, they would be required to respond in a manner that was consistent with their own beliefs and sometimes in a manner that was inconsistent with their beliefs.

\subsection{General procedure}

Following informed consent, participants completed either the behavioral approach tasks or the IRAPs first. The procedure was 
Table 1

Implicit-explicit/behavioural correlation matrix.

\begin{tabular}{|c|c|c|c|c|c|c|c|c|c|c|c|}
\hline & DP & DS & BAT & DS-R & OCI-R & Depr & Anx & Stress & DASS & Obsessing & Washing \\
\hline DP-IRAP & - & .265 & .091 & .049 & $.400^{*}$ & $.413^{*}$ & .230 & .104 & .312 & $.494^{* *}$ & .234 \\
\hline DS-IRAP & & - & $-.473^{* *}$ & .237 & $.406^{*}$ & $.449^{*}$ & .267 & .252 & $.400^{*}$ & .318 & $.391^{*}$ \\
\hline BAT & & & - & -.341 & -.369 & .005 & -.273 & -.004 & -.104 & -.160 & -.277 \\
\hline DS-R & & & & - & .248 & .009 & .213 & .081 & .117 & .282 & .143 \\
\hline OCI-R & & & & & - & $.443^{*}$ & .343 & .371 & $.473^{* *}$ & $.764^{* * *}$ & $.701^{* * *}$ \\
\hline Depression & & & & & & - & .345 & $.570^{* *}$ & $.799 * * *$ & .281 & $.447^{*}$ \\
\hline Anxiety & & & & & & & - & $.630^{* * *}$ & $.782^{* * *}$ & $.537^{* * *}$ & .161 \\
\hline Stress & & & & & & & & - & $.885^{* * *}$ & $.522^{* *}$ & .297 \\
\hline DASS & & & & & & & & & - & $.534^{* *}$ & $.376^{*}$ \\
\hline Obsessing & & & & & & & & & & - & $.463^{* *}$ \\
\hline
\end{tabular}

${ }^{*} p<.05$.

${ }^{* *} p<.01$.

${ }^{* * *} p<.001$.

counterbalanced so that half of the participants received the behavioral approach tasks first and the other half received the two IRAPs first. The order in which the behavioral approach tasks were administered was randomized to avoid obtaining a result that was specific to receiving the BATs in a particular sequence. The order in which the IRAPs were administered was counterbalanced. The questionnaires (DS-R, OCI-R, DASS) were administered between completion of the two IRAPs. Participants were given the opportunity to wash their hands with an antibacterial hand gel between the behavioral approach tasks to reduce residual disgust from one task affecting performance on the next task.

\section{Results}

\subsection{Scoring the IRAP}

To insure that the analyzed data reflected the relational stimulus control targeted by the IRAP, participants' accuracy scores for each block were first screened. Specifically, if response accuracy fell below $75 \%$ on only one test block then participant's analyses were conducted on the remaining two pairs of test blocks; the data for 1 participant were analyzed in this way. ${ }^{5}$ If a participant failed to maintain $\geq 75 \%$ across two or more test blocks, their entire data set for both IRAPs was discarded; the datasets for 4 participants were removed on this basis. The data from three additional participants were removed; for two outliers (for one the D-IRAP score was $2 S D$ s from the mean scores on the DP-IRAP, and for the other the OCI-R and DASS scores were 3 SDs from the mean) and for one participant who chose not to complete any of the BATs.

Using an adapted form of the Greenwald, Nosek, and Banaji (2003) $D$-algorithm, the response latencies were transformed into $D$-IRAP scores (for a full description see Vahey, Barnes-Holmes, Barnes-Holmes, \& Stewart, 2009; Nicholson \& Barnes-Holmes, in press).

\subsection{Internal consistency of socio-moral scale}

The socio-moral scale designed for the current study was found to have good internal consistency with a Cronbach's alpha of .87 . The inter-item correlations (the items measured being the number of scenarios written down, reported level of discomfort, willingness and difficulty: See Appendix A) were good-excellent, ranging from .49 to .84 , suggesting that the scales were related without being redundant. The positive correlations between the number of

\footnotetext{
${ }^{5}$ An accuracy criterion of $75 \%$, rather than the more typical $80 \%$, was implemented to avoid excessively high attrition rates (Vahey, Barnes-Holmes, BarnesHolmes, \& Stweart, 2009)
}

scenarios and the level of discomfort suggest that thinking and writing immoral scenarios evoked discomfort in the participants.

\subsection{Scoring the BATs}

Each behavioral task was scored on a six-point scale. To provide one overall score for the BATs the scores from the four disgustrelated tasks were added and then divided by four to obtain an average BAT score.

\subsection{Implicit measure analyses}

As noted in the Introduction, a significant limitation of the IAT is that it provides a relative response bias to two concepts, rather than a non-relative bias toward a single concept (De Houwer, 2002). In contrast, each trial-type may be analyzed separately with the IRAP (e.g., how distressing does an individual find disgusting things). In relation to the current research, the extent to which an individual finds disgusting stimuli as being positive, or pleasant stimuli as being negative, is somewhat irrelevant given that the problematic behavior of an individual with contamination-related OCD arises from an irrational interpretation of specific stimuli as being dangerous or distressing. Thus, the current set of analyses focused only on the IRAP trial-types that aimed to assess response biases that reflected negative reactions to disgust-eliciting stimuli (i.e., the Disgust-Bad and Disgust-Distressing trial-types). ${ }^{6}$ Both of these scores were consistent with the predicted response biases; that is, responding True more quickly than False to disgusting images paired with negative descriptors (Disgust-Bad, $M=.23, S D=.26$; Disgust-Distressing, $M=.17, S D=.35)$. Both scores proved to be significantly different from zero: Disgust-Bad, $t(25)=4.43$, $p=.0002 ;$ Disgust-Distressing, $t(25)=2.53, p=.01$.

\subsection{Correlation analyses}

A correlation matrix was calculated to examine the relationships between $D$-IRAP scores on the both the DP- and DS-IRAPs, overall performance on the BATs, general disgust (as measured by the DS$\mathrm{R})$, general psychopathology (as measured by the DASS), depression, anxiety and stress (also measured by the DASS), and OC tendencies (including obsessing and washing, as measured by the OCI-R). The results indicated that stronger disgust responses on the Disgust-Bad trial-type predicted higher levels of OC tendencies and

\footnotetext{
${ }^{6}$ The $D$-IRAP scores for the other trial-types were in the expected direction and in keeping with the hypothesis that participants' will have shorter response latencies on trials that require them to respond that disgusting things are bad/not good and that pleasant things are good/not bad.
} 
Table 2

Results from twelve hierarchical multiple regression analyses using the Disgust-Bad and the Disgust-Distressing trial-types predicting behavior, general OC tendencies and its sub-components while controlling for the effects of the other trial-type and anxiety.

\begin{tabular}{|c|c|c|c|c|c|}
\hline & & $B$ & SE $B$ & Beta & $p$ \\
\hline \multicolumn{6}{|c|}{ Dependent Variable: Behavior } \\
\hline \multirow[t]{3}{*}{1.} & Step $1\left(R^{2}=.008\right)$ & & & & \\
\hline & Disgust-Bad trial-type & .291 & .653 & .091 & .660 \\
\hline & Step $2\left(R^{2}\right.$ change $\left.=.265\right)$ & & & & \\
\hline \multirow{4}{*}{2.} & Disgust-Distressing trial-type & -1.33 & .459 & -.543 & .008 \\
\hline & Step $1\left(R^{2}=.074\right)$ & & & & \\
\hline & Anxiety & -.029 & .021 & -.273 & .178 \\
\hline & Step $2\left(R^{2}\right.$ change $\left.=.172\right)$ & & & & \\
\hline \multirow{2}{*}{\multicolumn{2}{|c|}{$\begin{array}{l}\text { Disgust-Distressing trial-type } \\
\text { Dependent Variable: OC Tendencies }\end{array}$}} & -1.07 & .467 & -.431 & .031 \\
\hline & & & & & \\
\hline \multirow[t]{4}{*}{3.} & Step $1\left(R^{2}=.061\right)$ & & & & \\
\hline & DS-R & .265 & .212 & .248 & .223 \\
\hline & Step $2\left(R^{2}\right.$ change $\left.=.151\right)$ & & & & \\
\hline & Disgust-Bad trial-type & 14.47 & 6.90 & .388 & .047 \\
\hline \multirow[t]{3}{*}{4.} & Step $1\left(R^{2}=.164\right)$ & & & & \\
\hline & $\begin{array}{l}\text { Disgust-Distressing trial-type } \\
\text { Step } 2\left(R^{2} \text { change }=.092\right)\end{array}$ & 11.70 & 5.30 & .406 & .040 \\
\hline & Disgust-Bad trial-type & 11.70 & 6.89 & .314 & .105 \\
\hline \multirow[t]{4}{*}{5.} & Step $1\left(R^{2}=.118\right)$ & & & & \\
\hline & Anxiety & .430 & .240 & .343 & .080 \\
\hline & Step $2\left(R^{2}\right.$ change $\left.=.109\right)$ & & & & \\
\hline & Disgust-Bad trial-type & 12.61 & 7.02 & .339 & .085 \\
\hline \multirow[t]{4}{*}{6.} & Step $1\left(R^{2}=.061\right)$ & & & & \\
\hline & DS-R & .265 & .212 & .248 & .223 \\
\hline & Step $2\left(R^{2}\right.$ change $\left.=.127\right)$ & & & & \\
\hline & Disgust-Distressing trial-type & 10.60 & 5.58 & .367 & .070 \\
\hline \multirow[t]{4}{*}{7.} & Step $1\left(R^{2}=.160\right)$ & & & & \\
\hline & Disgust-Bad trial-type & 14.88 & 6.97 & .400 & .043 \\
\hline & Step $2\left(R^{2}\right.$ change $\left.=.097\right)$ & & & & \\
\hline & Disgust-Distressing trial-type & 9.30 & 5.38 & .322 & .097 \\
\hline \multirow[t]{4}{*}{8.} & Step $1\left(R^{2}=.118\right)$ & & & & \\
\hline & Anxiety & .430 & .240 & .343 & .086 \\
\hline & Step $2\left(R^{2}\right.$ change $\left.=.106\right)$ & & & & \\
\hline & Disgust-Distressing trial-type & 9.76 & 5.50 & .338 & .089 \\
\hline \multicolumn{6}{|c|}{ Dependent Variable: Obsessing } \\
\hline \multirow[t]{4}{*}{9.} & Step $1\left(R^{2}=.101\right)$ & & & & \\
\hline & Disgust-Distressing trial-type & 2.39 & 1.45 & .318 & .113 \\
\hline & Step $2\left(R^{2}\right.$ change $\left.=.181\right)$ & & & & \\
\hline & Disgust-Bad trial-type & 4.26 & 1.77 & .441 & .025 \\
\hline \multirow[t]{4}{*}{10.} & Step $1\left(R^{2}=.288\right)$ & & & & \\
\hline & Anxiety & .174 & .056 & .537 & .005 \\
\hline & Step $2\left(R^{2}\right.$ change $\left.=.145\right)$ & & & & \\
\hline & Disgust-Bad trial-type & 3.78 & 1.56 & .391 & .023 \\
\hline \multicolumn{6}{|c|}{ Dependent Variable: Washing Concerns } \\
\hline \multirow[t]{4}{*}{11.} & Step $1\left(R^{2}=.055\right)$ & & & & \\
\hline & Disgust-Bad trial-type & 2.09 & 1.77 & .234 & .251 \\
\hline & Step $2\left(R^{2}\right.$ change $\left.=.116\right)$ & & & & \\
\hline & Disgust-Distressing trial-type & 2.45 & 1.63 & .354 & .086 \\
\hline \multirow[t]{4}{*}{12.} & Step $1\left(R^{2}=.026\right)$ & & & & \\
\hline & Anxiety & .045 & .060 & .161 & .432 \\
\hline & Step $2\left(R^{2}\right.$ change $\left.=.130\right)$ & & & & \\
\hline & Disgust-Distressing trial-type & 2.59 & 1.38 & .374 & .072 \\
\hline
\end{tabular}

specifically obsessing along with depressive symptoms. Stronger IRAP effects on the Disgust-Distressing trial-type predicted fewer steps completed across the BATs, higher OC and depressive symptoms along with general psychopathology, and washing concerns specifically (see Table 1). Additional analyses indicated that the differences between these correlations were not significant, and thus caution is required in interpreting these results as strong evidence that the two IRAPs were tapping into separate constructs.

\subsection{Predictive validity of the IRAP}

Given the significant correlations, it was deemed important to conduct a series of hierarchical multiple regression analyses to determine the predictive validity of one $D$-IRAP trial-type independently of the other trial-type. In addition, based on suggestions in the literature regarding the study of the relationship between disgust and psychopathology (Davey, 2003), hierarchical multiple regression were subsequently used to control for the effects of anxiety.

In Table 2 it can be seen that the Disgust-Distressing trial-type predicted behavior independently of the Disgust-Bad trial-type and anxiety. Additionally, neither trial-type remained a significant predictor of OC tendencies when the other trial-type or anxiety was controlled for. Though, the Disgust-Bad trial-type did remain a significant predictor of $\mathrm{OC}$ tendencies when self-reported disgust was controlled for while the Disgust-Distressing trial-type was marginally so. Only the Disgust-Bad trial-type remained a significant predictor of OC tendencies when the DS-R was controlled for, however, the Disgust-Distressing trial-type was marginally significant. Finally, neither the Disgust-Distressing trialtype nor anxiety undermined the predictive validity of the Disgust-Bad trial-type for obsessing, while the predictive validity of the Disgust-Distressing trial-type only remained significant for washing concerns when controlling for anxiety and marginally so when controlling for the Disgust-Bad trial-type.

\section{Discussion}

The current study sought to determine if disgust propensity and sensitivity could be individually assessed by a measure of implicit cognition, the IRAP. The results provided preliminary evidence that the two IRAPs were measuring two separate constructs. For instance, the IRAP measuring disgust propensity did not predict behavior during the BATs while the IRAP measuring disgust sensitivity did. Implicit disgust propensity and sensitivity predicted $\mathrm{OC}$ tendencies, but only disgust propensity acted as a predictor of the obsession subscale of the OCI-R, while disgust sensitivity had a relationship with the washing subscale of the OCI-R. There was a non-significant positive correlation (.26) between the DP-IRAP and the DS-R which suggests a relationship between these variables - the relatively small $\mathrm{N}$ may account for the lack of statistical significance. Interestingly, both IRAPs were related to depressive symptoms, however, as this is not the sole concern of this paper it will not be discussed further.

In addition, the impact of each trial-type on the predictive validity of the other was assessed. That is, when you control for the initial feeling of disgust (propensity), is the secondary appraisal of disgust (sensitivity) capable of predicting behavior/OC tendencies and vice-versa. In terms of behavior, this was found to be true in that disgust sensitivity was a significant predictor of avoidance when the effects of both disgust propensity and anxiety were controlled for. On the other hand, in relation to OC tendencies, neither trial-type were significant predictors when the other was controlled for (although both approached significance). A possible explanation for this effect could be that each variable accounts for different aspects of OCD, as evident in the subsequent analyses that showed specific predictive effects for obsessing (with disgust propensity) and washing concerns (with disgust sensitivity).

The present results support the proposition that disgust responding occurs at both a primary (disgust propensity) and secondary (disgust sensitivity) level (van Overveld et al., 2006). Teachman and Saporito (2009) observed evidence of both primary and secondary disgust cognitions in relation to spider fear and Blood-Injury-Injection (BII) phobia with a series of behavioral approach tasks. van Overveld et al. (2006) argued that failing to acknowledge both disgust propensity and disgust sensitivity could lead to inflated correlations between disgust and behavior in experimental settings. If disgust is to be implicated in the etiology of OCD, measures which are not confounded by such restrictions 
are vital. Thus, the current study provides two measures of disgust which assess distinctive aspects of responding and demonstrate their individual relationships with psychopathology. Moreover, this is the first study to demonstrate such sensitivity with a measure of implicit cognition, the IRAP.

The use of implicit measures, it has been argued, may provide insights into the aspects of psychopathology which can appear irrational and uncontrollable (Wiers et al., 2007). For example, it is unclear if people suffering from conditions such as OCD have conscious access to the mechanisms controlling irrational behaviors and if these mechanisms can be measured through self-report means. For instance, one individual with a diagnosis of OCD and another without may both overtly classify something as being equally disgusting (e.g., on a scale of one to ten), but the individual with a diagnosis of OCD may be experiencing a greater amount of disgust. Indeed, in the current study there was a non-significant positive relationship between scores on the DS-R and OC tendencies, while implicit disgust propensity and sensitivity both correlated significantly with OC tendencies. Thus, it appears that the implicit measure detected variations in disgust propensity and sensitivity where the self-report measure failed to do so. However, it should be noted that previous studies have found a significant relationship between self-reported disgust propensity and $\mathrm{OC}$ tendencies (Berle et al., 2012). Indeed, the present results obtained a similar correlation $(r=.24)$ to the Berle et al. (2012) study, but in the current case the $N$ was approximately four times less, thus probably accounting for the lack of significance at the .05 level. In any case, the present results support the use of implicit measures in the study of psychopathology.

The finding that disgust sensitivity predicted avoidance behavior on the BATs while disgust propensity did not suggests that the initial feeling of disgust may not be indicative of behavior. Rather, it appears that it is the appraisal of disgust which results in behavioral avoidance. This evidence provides empirical support for Teachman's (2006) theory of pathological disgust, who hypothesized that primary responses to disgust-eliciting stimuli would focus on beliefs regarding the likelihood of contamination or becoming disgusted (e.g., "It turns my stomach" or "Will contaminate me"), whereas secondary appraisals or interpretations would reflect the individual's perceived ability to cope with the initial feeling of disgust (e.g., "I worry I'll get sick" or "I cannot tolerate it"). Thus, it is presumed that while the majority of individuals would experience some degree of disgust in response to a disgust-eliciting stimulus, those who interpret this response as being threatening or meaningful are more likely to engage in avoidance behavior. As an aside, previous studies using implicit measures to assess disgust in relation to spider fear (e.g., Teachman \& Woody, 2003) have found that disgust propensity-like-characteristics predicted behavior. However, these studies were measuring disgust in relation to spider fear not disgust as a whole. Thus, it is difficult to compare the current findings directly with the previous research on disgust that focused specifically on spiders.

The current findings may also be relevant to the seminal work of Salkovskis (1989) on the cognitive-behavioral aspect of obsessions. Specifically, he asserted that intrusive thoughts carry no specific tone until they are positively, negatively or neutrally appraised by an individual. It was further posited that the overt or covert behavioral reactions to the initial intrusive cognition will become salient to the individual. When this appraisal has direct implications of possible harm, it will result in discomfort which must be neutralized (e.g., through hand-washing). Once the neutralizing responses to the intrusive thoughts are established, they are preserved through an association with less discomfort. The fact that disgust propensity predicted obsessions and sensitivity predicted avoidance and washing concerns could be seen as generally consistent with Salkovskis' argument. That is, if intrusive thoughts constitute an important component of disgust propensity, and sensitivity is an important component of the appraisal of such thoughts, this would explain the relationship between propensity and self-reported obsessions on the one hand and sensitivity and actual behavioral avoidance on the other.

It should be noted that while disgust propensity did not predict avoidance behavior it did predict OC tendencies along with disgust sensitivity. Thus, OCD populations may have a stronger propensity to become disgusted than a normative population. However the extent of these differences has yet to be established. For instance, disgust-related intrusive thoughts may simply be more salient to a person with a diagnosis of OCD, which increases propensity, or they may actually experience a greater number of intrusive thoughts. It is worth noting, however, that the relationship between disgust propensity and $\mathrm{OC}$ tendencies in the current study was influenced by anxiety, which suggests that trait anxiety may exacerbate the initial experience of disgust (Davey, 2003).

At this point, it should be acknowledged that one limitation of the current study is that the obsessions subscale of the OCI-R measures general obsessing, rather than specific obsessions (e.g., contamination, checking, etc.), and thus the specific relationship between disgust propensity and specific obsessions remains unclear. Perhaps, for example, obsessions related to contamination, rather than checking, are better predicted by disgust propensity. Further research will thus be needed to examine the specific obsessions predicted by disgust propensity.

The finding that disgust sensitivity predicts washing concerns above and beyond anxiety expands on research by Olatunji et al. (2007), who found that both anxiety and general disgust were predictors of washing concerns. The authors concluded that disgust acts a significant intervening variable, in that the path from anxiety to washing concerns decreased when disgust was added to a regression model. However, the study conceptualized disgust as a unitary response, and thus it may have been affected by an interaction between propensity and sensitivity, as predicted by van Overveld et al. (2006), which may have weakened the role played by disgust. Indeed, the current findings indicate that disgust sensitivity may be the factor through which disgust responding becomes pathological above and beyond the impact of anxiety.

A general limitation of the present study was the use of a nonclinical student population from a particular cultural background (i.e., Irish). On balance, there is little evidence that a cultural variable affects concurrent levels of disgust propensity or sensitivity (Oaten et al., 2009). Furthermore, there is evidence that OCD responding can be recorded in non-patient samples (Burns et al., 1995). Nevertheless, extending the current study by comparing a non-clinical sample who score high on a self-report measure of OCD and a sample of patients with a diagnosis of OCD would provide further insight into the relationship between disgust responding and OCD.

The two IRAPs in the current study presented identical disgusteliciting photographs while the target stimuli changed depending on which aspect of disgust the IRAP was designed to measure. The finding that two distinct yet very similar IRAPs produced converse results has important implications for the IRAP itself. Firstly, it emphasizes the importance of the target stimuli in the IRAP. Future researchers should thus take great care to select stimuli which are meaningful and relevant to the theory which underpins the questions under consideration. Secondly, it demonstrates the ability of the IRAP to measure the subtle differences which encompass one type of responding. This shows the flexibility of the IRAP as a measure of different aspects of psychopathology. In closing, it is worth noting that the current study examined disgust responding across a range of disgust domains, but future research could 
determine if even greater specificity is possible by attempting to measure implicit disgust propensity and sensitivity in relation to each specific domain of disgust (e.g., body-envelope violations, socio-moral etc).

\section{Acknowledgments}

The research and preparation for this article was supported by the John and Pat Hume Scholarship Scheme awarded to the first author.

We would like to thank Bethany Teachman who provided constructive and helpful comments on an earlier draft of this article.

\section{Appendix A. Socio-Moral Scale}

Instructions: Please think of three examples or scenarios of a moral violation which you would consider to be weak/ moderate/strong/extreme violations of morality.

Please make sure you stop if you feel uncomfortable or find that you cannot think of three scenarios.

Example of a weak violation of morality. A women is cleaning out her closet, and she finds her old Irish flag. She doesn't want the flag anymore, so she cuts it up into pieces and uses the rags to clean her bathroom.

\section{Weak/Moderate/Strong/Extreme Moral Violation 1}

\section{Weak/Moderate/Strong/Extreme Moral Violation 2.}

Weak/Moderate/Strong/Extreme Moral Violation 3.

Please answer the following questions based on the scales provided

1. How difficult did you find this task?

\begin{tabular}{|l|l|l|l|l|l|l|l|l|l|}
\hline 1 & 2 & 3 & 4 & 5 & 6 & 7 & 8 & 9 & 10 \\
\hline
\end{tabular}

\section{( 1 being extremely easy and 10 being extremely difficult)}

2. How morally uncomfortable did you feel when carrying out this challenge?

\begin{tabular}{|l|l|l|l|l|l|l|l|l|l|}
\hline 1 & 2 & 3 & 4 & 5 & 6 & 7 & 8 & 9 & 10 \\
\hline
\end{tabular}

( 1 being extremely comfortable and 10 being extremely uncomfortable)

3. How willing would you be to do this task again?

\begin{tabular}{|l|l|l|l|l|l|l|l|l|l|}
\hline 1 & 2 & 3 & 4 & 5 & 6 & 7 & 8 & 9 & 10 \\
\hline
\end{tabular}

( 1 being extremely willing and 10 being extremely unwilling) NB: You should consider stopping with the task if you answered 8 or above on No. 3.

\section{References}

Barnes-Holmes, D., Barnes-Holmes, Y., Power, P., Hayden, E., Milne, R., \& Stewart, I. (2006). Do you really know what you believe? Developing the Implicit Relational Assessment Procedure (IRAP) as a direct measure of implicit beliefs. The Irish Psychologist, 32, 169-177.

Barnes-Holmes, D., Barnes-Holmes, Y., Stewart, I., \& Boles, S. (2010). A sketch of the Implicit Relational Assessment Procedure (IRAP) and the Relational Elaboration and Coherence (REC) model. The Psychological Record, 60, 527-542.

Berle, D., Starcevicb, V., Brakouliasb, V., Sammutc, P., Milicevica, D., Hannana, A. et al. (2012). Disgust-propensity in obsessive-compulsive disorder: cross- sectional and prospective relationships. Journal of Behavior Therapy and Experimental Psychiatry, 43, 656-663.

Burns, G. L., Formea, C. L., Koertege, S., \& Sternberger, L. G. (1995). The utilisation of non-patient samples in the study of obsessive compulsive disorder. Behaviour Research and Therapy, 33, 133-144.

Davey, G. C. L. (2003). What is interesting isn't always useful. The Psychologist, 16 , $412-416$.

Davey, G. C. L., MacDonald, B. A., \& Brierley, L. (2008). The effect of disgust on anxiety ratings to fear-relevant, disgust-relevant, and fear-irrelevant stimuli. Journal of Anxiety Disorders, 22, 1347-1354.

De Houwer, J. (2002). The Implicit Association Test as a tool for studying dysfunctional associations in psychopathology: strengths and limitations. Journal of Behavior Therapy and Experimental Psychiatry, 33, 115-133.

De Houwer, J. (2003). The extrinsic affective Simon task. Experimental Psychology, $50,77-85$.

Foa, E.,B., Huppert, J. D., Leiberg, S., Langner, R., Kichic, R., Hajcak, G., et al. (2002) The obsessive-compulsive inventory: development and validation of a short version. Psychological Assessment, 14, 485-496.

Gordan, T. L., \& Teachman, B. A. (2008). Ethnic differences in affective, behavioral, and cognitive markers of anxiety. Journal of Cross-Cultural Psychology, 39, 424-446.

Greenwald, A. G., Nosek, B. A., \& Banaji, M. R. (2003). Understanding and using the implicit association test: I. An improved scoring algorithm. Journal of Personality and Social Psychology, 85, 197-216.

Haidt, J., McCauley, C., \& Rozin, P. (1994). Individual differences in sensitivity to disgust: a scale sampling seven domains of disgust elicitors. Personality and Individual Differences, 16, 701-713.

Hayes, S. C., Barnes-Holmes, D., \& Roche, B. (2001). Relational frame theory: A postSkinnerian account of human language and cognition. New York: Kluwer/Plenum.

Henry, J. D., \& Crawford, J. R. (2005). The short-form version of the Depression Anxiety Stress Scales (DASS-21): construct validity and normative data in a large non-clinical sample. British Journal of Clinical Psychology, 44, 227-239.

Hughes, S., Barnes-Holmes, D., \& De Houwer, J. (2011). The dominance of associative theorising in implicit attitude research: propositional and behavioral alternatives. The Psychological Record, 61, 465-496.

Huijding, J., \& de Jong, P. J. (2007). Beyond fear and disgust: the role of (automatic) contamination-related associations in spider phobia. Journal of Behavior Therapy and Experimental Psychiatry, 38, 200-211.

Lang, P. J., Bradley, M. M., \& Cuthbert, B. N. (1996). International Affective Picture System (IAPS): Technical manual and affective ratings. NIMHCenter for the Study of Emotion and Attention.

Lovibond, S. H., \& Lovibond, P. F. (1993). Manual for the Depression Anxiety Stress Scales (DASS). Psychology Foundation Monograph. (Available from The Psychology Foundation, Room 1005 Mathews Building, University of New SouthWales, NSW 2052, Australia).

McNally, R. J. (1995). Automaticity and the anxiety disorders. Behaviour Research and Therapy, 33, 747-754.

Moretz, M.w., \& McKay, D. (2008). Disgust sensitivity as a predictor of obsessivecompulsive contamination symptoms and associated cognitions. Journal of Anxiety Disorders, 22, 707-715.

Nicholson, E., \& Barnes-Holmes, D. The Implicit Relational Assessment Procedure (IRAP) as a measure of spider fear. The Psychological Record, in press.

Nosek, B. A., \& Greenwald, A. G. (2009). (Part of) the case for a pragmatic approach to validity: comment on De Houwer, Teige-Mocigemba, Spruyt, and Moors (2009). Psychological Bulletin, 135, 373-376.

Nosek, B. A., Hawkins, C. B., \& Frazier, R. S. (2011). Implicit social cognition: from measures to mechanisms. Trends in Cognitive Sciences, 15, 152-159.

Oaten, M., Stevenson, R. J., \& Case, T. I. (2009). Disgust as a disease-avoidance mechanism. Psychological Bulletin, 135, 303-321.

Olatunji, B. O., Cisler, J., McKay, D., \& Phillips, M. L. (2010). Is disgust associated with psychopathology? Emerging research in the anxiety disorders. Psychiatry Research, 175, 1-10.

Olatunji, B. O., Williams, N. L., Lohr, J. M., Connelly, K. M., Cisler, J., \& Meunier, S. A. (2007). Structural differentiation of disgust from trait anxiety in the prediction of specific anxiety disorder symptoms. Behaviour Research and Therapy, 45, 3002-3017.

van Overveld, W. J. M., de Jong, P. J., Peters, M. L., Cavanagh, K., \& Davey, G. C. L. (2006). Disgust propensity and disgust sensitivity: separate constructs that are differentially related to specific fears. Personality and Individual Differences, 41, 1241-1252.

van Overveld, W. J. M. de Jong P. J., Peters, M. L. \& Schouten, E. (2011). The Disgust Scale-R: a valid and reliable index to investigate separate disgust domains? Personality and Individual Differences, 51, 325-330.

Rozin, P., Haidt, J., McCauley, C., Dunlop, L., \& Ashmore, L. (1999). Individual differences in disgust sensitivity: comparisons and evaluations of paper-and-pencil and versus behavioural measures. Journal of Research in Personality, 33, 330-351.

Rozin, P., Millman, L., \& Nemeroff, C. (1986). Operation of the laws of sympathetic magic in disgust and other domains. Journal of Personality and Social Psychology, 50, 703-712.

Salkovskis, P. (1985). Obsessional compulsive problems: a cognitive behavioural analysis. Behaviour Research and Therapy, 23, 571-583.

Salkovskis, P. (1989). Cognitive-behavioural factors and the persistence of intrusive thoughts in obsessional problems. Behaviour Research and Therapy, 27, 677-682.

Shafran, R., \& Rachman, S. (2004). Thought-action fusion: a review. Journal of Behavior Therapy and Experimental Psychiatry, 35, 87-107.

Teachman, B. A. (2006). Pathological disgust: in the thoughts, not the eye, of the beholder. Anxiety, Stress, and Coping, 19, 335-351. 
Teachman, B. A. (2007). Evaluating implicit fear associations using the Go/No-go association task. Journal of Behavior Therapy and Experimental Psychiatry, 38, 156-167.

Teachman, B. A., Gregg, A. P., \& Woody, S. R. (2001). Implicit associations for fear relevant stimuli among individuals with snake and spider fears. Journal of Abnormal Psychology, 110, 226-235.

Teachman, B. A., \& Saporito, J. (2009). I am going to gag: disgust cognitions in spider and blood-injury-injection fears. Cognition and Emotion, 23, 399-414.

Teachman, B. A., \& Woody, S. R. (2003). Automatic processing in spider phobia: implicit fear associations over the course of treatment. Journal of Abnormal Psychology, 122, 100-109.

Vahey, N., Barnes-Holmes, D., Barnes-Holmes, Y., \& Stewart, I. (2009). A first test of the implicit relational assessment procedure as a measure of self-esteem: Irish prisoner groups and university students. The Psychological Record, 59, 371-388.

Wahl, K., Kordon, A., Kuelz, K. A., Voderholzer, U., Hohagen, F., \& Zurowski, B. (2010) Obsessive Compulsive Disorder (OCD) is still an unrecognised disorder: a study on the recognition of OCD in psychiatric outpatients. European Psychiatry, 25 374-377.

Wiers, R. W., Teachman, B. A., \& De Houwer, J. (2007). Implicit cognitive processes in psychopathology: an introduction. Journal of Behavior Therapy and Experimenta Psychiatry, 38, 95-104.

Zinkernagel, A., Hofman, W., Dislich, F. X. R., Gschwendner, T., \& Schmitt, M. (2011) Indirect assessment of implicit disgust sensitivity. European Journal of Psychological Assessment, 27, 237-243. 